\title{
Más allá del principio de proporcionalidad $^{* * * * * * *}$
}

\section{Beyond the principle of proportionality}

RESUMEN

El presente artículo aborda el principio de proporcionalidad a la luz de una teoría sustantiva de la justicia y los derechos subjetivos que lo fundamenta. A pesar de la existencia del principio de proporcionalidad, resulta necesaria una teoría "externa" a la mecánica del funcionamiento del principio de proporcionalidad. Una que sirva de soporte a los jueces al momento de responder las preguntas normativas que exige la aplicación del principio. Los pasos y las probabilidades de la proporcionalidad no son suficientes a la hora de identificar distinciones cualitativas entre derechos o atribuir pesos abtractos a la restricción de un derecho vis-à-vis el cumplimiento de un fin legítimo o la garantía de otro derecho de igual importancia. Por tal razón, el presente artículo subraya la necesidad de elaborar serie de teorías sustantivas que sustenten el test de proporcionalidad al determinar, entre otros elementos, el alcance y la aplicación de los derechos en las diferentes esferas sociales, el efecto horizontal de los derechos constitucionales en el ámbito privado, o las implicaciones institucionales de la aplicación del principio de constitucionalidad al determinar el rol de los tribunales en ejercicio del control constitucional.

Catedrático de Derecho Constitucional de la Universidad Pompeu Fabra. Máster en Derecho (LL.M) y Doctor en Derecho (JSD), Yale Law School. Licenciado en Derecho, Universidad de Barcelona.

** Recibido el 8 de octubre del 2019, aprobado el 28 de enero del 2020.

Para citar el artículo: FerReres Comella V. Más allá del principio de proporcionalidad. En Revista Derecho del Estado, Universidad Externado de Colombia. N. . 46, mayo-agosto de 2020, pp. 161-188.

DOI: https://doi.org/10.18601/01229893.n46.07

**** Texto original en inglés en: Ferreres Comella, Víctor. Beyond the principle of proportionality. En JACOBSOHN, GARY Y SCHOR, Miguel (EDS.). Comparative Constitutional Theory. Cheltenham: Edward Elgar Publishing, 2018. Traducción por Marcelo Lozada Gómez y María Camila Medina García, docentes de derecho constitucional, Universidad Externado de Colombia. 
PALABRAS CLAVE

Proporcionalidad, ponderación, derechos fundamentales, teoría sustantiva de los derechos.

ABSTRACT

In this article, the very essence of proportionality is assessed in light of a substantive theory of justice and rights. Despite the existence of the principle of proportionality, a theory that is "external" to the mechanics of proportionality is deemed necessary. One that facilitates the work of judges when assessing the normative issues when they adjudicate using the principle of proportionality. The steps and probabilities of proportionality are not enough in order to draw qualitative distinctions among rights or balance the abstract weight of the right's limitation vis-à-vis the achievement of legitimate ends or the guarantee of other rights. Therefore, this article underscores the need of background substantive theories that underpin the application of the principle. Such theories should address, among other issues, the scope and enforcement of rights in the different spheres of social life, the horizontal effect of constitutional rights in the private domain, or the institutional consequences of the principle concerning the role of courts when exercising constitutional review.

\section{KEYWORDS}

Proportionality, balancing, fundamental rights, horizontal effect.

\section{SUMARIO}

Introducción. I. Delimitar derechos absolutos y derechos relativos. A. Carácter absoluto e importancia del derecho. B. Carácter absoluto y cláusula de intangibilidad. C. Carácter absoluto y suspensión de derechos. D. Identificar derechos absolutos: las limitaciones del lenguaje constitucional. II. Identificación de fines legítimos. III. Ponderación (balancing) y el problema de los pesos abstractos IV. Establecer diferencias cualitativas dentro del campo de operación de un derecho. V. Proporcionalidad y el efecto horizontal de los derechos fundamentales. VI. Más allá de la proporcionalidad: algunas consecuencias institucionales. Referencias.

\section{INTRODUCCIÓN}

El principio de proporcionalidad es un marco doctrinal que las cortes en muchas jurisdicciones usan para determinar si una restricción particular de un derecho fundamental adoptada por el Gobierno está justificada a la luz de 
la constitución. En su forma más común, este principio se utiliza en un test de tres elementos o pruebas. Cada paso plantea una pregunta que requiere ser contestada de forma afirmativa para que las cortes puedan concluir que la limitación del derecho bajo examen está justificada. Los tres pasos son: en primer lugar, ¿es la restricción una medida idónea para alcanzar, o ayudar a lograr, un objetivo constitucional legítimo? En segundo lugar, ¿es esta restricción realmente necesaria? En otros términos, ¿existe alguna medida menos invasiva del derecho que permita alcanzar el mismo objetivo? En tercer lugar, ¿es la carga impuesta al ejercicio del derecho proporcional al beneficio que se deriva de ella en términos de la consecución del objetivo constitucional? $\mathrm{Si}$ una medida proferida por el gobierno satisface estas tres exigencias (las cuales usualmente son llamadas idoneidad, necesidad y proporcionalidad en sentido estricto o ponderación), la medida será convalidada por los jueces.

El principio de proporcionalidad, concebido de esta forma, fue desarrollada en Alemania, en vigencia de la ley fundamental de Bonn de 1949. Sin embargo, sus orígenes se remontan al derecho administrativo decimonónico. Las cortes administrativas alemanas, especialmente el Obertverwaltungsgericht prusiano, recurrieron de forma constante a análisis de proporcionalidad para limitar los poderes de policía del Estado ${ }^{1}$. A partir de allí, otros países en todo el mundo incorporaron este instrumento dentro de sus prácticas de decisión constitucional ${ }^{2}$. Existen, naturalmente, divergencias en la forma en que este principio ha sido estructurado y aplicado en distintas jurisdicciones ${ }^{3}$. Sin embargo, un marco común ha surgido.

Curiosamente, a pesar de la importancia práctica que ha adquirido el principio de proporcionalidad, pocas constituciones lo prevén explícitamente ${ }^{4}$. La constitución alemana, concretamente, no lo prevé. La proporcionalidad es el producto normativo de la jurisprudencia de las cortes y el trabajo doctrinal de académicos expertos. En particular, los filósofos del derecho han prestado mucha atención al principio de proporcionalidad, que ellos han atado a

1 Moshe CoHEn-Eliya e Iddo Porat, American Balancing and German Proportionality: The Historical Origins, 8 International Journal of Constitutional Law 263, 271-76 (2010).

2 Para una descripción detallada del origen y la evolución del principio de proporcionalidad en diferentes regiones del mundo, ver: Aharon Barak, Proportionality. Constitutional Rights and their Limitations 175-210 (2012). El libro incluye un mapeo útil que evidencia la migración del principio de proporcionalidad, Id. 182. Ver, a su vez, Alec Stone Sweet y Jud Mathews, Proportionality Balancing and Global Constitutionalism, 47 Columbia Journal of Transnational Law 72 (2008).

3 Respecto de las diferencias entre Canadá y Alemania ver, entre otros, Dieter Grimm, Proportionality in Canadian and German Constitutional Jurisprudence, 57 University of Toronto Law Journal 383, 2007. Como afirma Grimm, el tercer paso del análisis de proporcionalidad (ponderación) está menos presente en Canadá que en Alemania. Las cortes canadienses normalmente abordan dentro del componente de necesidad muchos elementos que las cortes alemanas prefieren analizar en este tercer paso del juicio de proporcionalidad.

$4 \quad$ Las constituciones de Rumania (artículo 53(2)), Turquía (artículo 13) y Suiza (art. 36(3)), por mencionar tan solo algunos ejemplos, sí incorporan explícitamente el principio. 
nociones básicas de justicia y razón práctica. Sin lugar a dudas, uno de los trabajos más influyentes en este ámbito es el de Robert Alexy ${ }^{5}$.

De forma similar, a nivel internacional y supranacional, tribunales y otros órganos a cargo de la implementación de instrumentos de derechos humanos recurren al principio de proporcionalidad al resolver casos particulares. Estos instrumentos, sin embargo, rara vez mencionan el principio de proporcionalidad. La Declaración Universal de Derechos Humanos, el Pacto Internacional de Derechos Civiles y Políticos, el Pacto Internacional de Derechos Económicos, Sociales y Culturales, y el Convenio Europeo de Derechos Humanos, por ejemplo, no contienen ninguna referencia expresa al principio de proporcionalidad. La más reciente Carta de Derechos Fundamentales de la Unión Europea, en contraste, sí contiene una referencia explícita a este principio 6 .

No es exagerado afirmar que la proporcionalidad se ha convertido en parte de la gramática constitucional de los derechos a nivel global. "Vivimos ahora en la era de la proporcionalidad" señala Aharon Barak7. "La decisión sobre derechos basada en la proporcionalidad", en palabras de Alec Stone Sweet y Jud Mathews, "constituye una de las características determinantes del constitucionalismo global".

Existe un amplio debate sobre la consolidación de Estados Unidos como una excepción a esta tendencia generalizada. Es posible determinar con claridad diferencias entre las doctrinas del constitucionalismo estadounidense y aquellas doctrinas desarrolladas en otros países. En particular, el tercer paso del test de proporcionalidad (proporcionalidad en sentido estricto) está menos presente en la jurisprudencia estadounidense que en otras jurisdicciones. Sin embargo, persiste la idea principal de que los derechos constitucionales pueden ser limitados en ocasiones por el gobierno, y que esas limitaciones están justificadas cuando están encaminados a la garantía de fines constitucionales significativos ${ }^{9}$. De hecho, el auge del pragmatismo jurídico en los años 30

5 Ver Robert Alexy, A Theory of Constitutional Rights (2002). Un análisis de este libro que usualmente sitúa la teoría de Alexy en el contexto de las discusiones actuales sobre derechos puede encontrarse en Mattias Kumm, Constitutional Rights as Principles: On the Structure and Domain of Constitutional Justice, 2 International Journal of Constitutional Law 574 (2004).

6 Ver la Carta de Derechos Fundamentales de la Unión Europea, 2000 O.J. (C 364), artículo 52 parágrafo 1 .

7 BARAK, supra nota 2, p. 457.

8 Stone SweEt y Mathews, supra nota 2, p. 74.

9 En Estados Unidos de América, los derechos usualmente se clasifican en tres grupos, cada uno de los cuales amerita un nivel distinto de escrutinio judicial (estricto, intermedio, mínimo). La restricción de un derecho puede estar o no justificada, dependiendo de la importancia del interés que el Gobierno desea promover y el tipo de balance que resulta de la restricción y el objetivo deseado. Cuanto mayor sea el nivel de escrutinio judicial debido, mayor deberá ser el carácter apremiante del objetivo promovido por el gobierno, y más estrecha la relación entre la medida restrictiva y el objetivo perseguido que justifica la restricción del derecho. En el derecho estadounidense, normalmente no existe ponderación ad hoc en esta tercera etapa del análisis. 
del siglo pasado abrió la puerta al denominado constitutional balancing, o balance constitucional de intereses ${ }^{10}$. La razón por la cual las cortes y tribunales de los Estados Unidos no han construido una doctrina general de la proporcionalidad está relacionada con aspectos contingentes del sistema jurídico estadounidense, los cuales han sido explorados por Vicki Jackson en un artículo reciente ${ }^{11}$.

El principio de proporcionalidad es aplaudido por muchos académicos y jueces correctamente. Su estructura de tres etapas permite introducir un cierto grado de disciplina intelectual en el razonamiento que las cortes desarrollan al evaluar limitaciones de derechos fundamentales. Además, el análisis de proporcionalidad puede ser visto como parte esencial de una "cultura de la justificación" en un contexto democrático. Individuos cuyo interés es lesionado por una ley pueden acudir a las cortes para requerir del gobierno una justificación de sus acciones con base en razones sustantivas aceptables. Este derecho a impugnar una ley es propiciado por una concepción amplia del ámbito de aplicación de los derechos constitucionalmente protegidos. Un entendimiento más amplio del ámbito de la justicia constitucional trae consigo mayores posibilidades de impugnar medidas gubernamentales ante las cortes ${ }^{12}$. Al mismo tiempo, el principio de proporcionalidad permite a los

En cambio, el peso atribuido al derecho y el peso del interés gubernamental son tomados en cuenta en un nivel más abstracto, como parte de la primera etapa del análisis, cuando las cortes clasifican los derechos y examina la legitimidad de los objetivos perseguidos por el gobierno. Una valoración de la jurisprudencia estadounidense desde una perspectiva comparada puede encontrarse en Barak, supra nota 2,pp. 509-521. Una postura interesante conforme a la cual la jurisprudencia estadounidense no es tan "excepcional" como usualmente se afirma, puede verse en Stephen Gardbaum, The Myth and the Reality of American Constitutional Exceptionalism, 107 Michigan Law Review 391 (2008). La principal diferencia, observa Gardbaum, es que la adjudicación constitucional en torno a derechos en Estados Unidos normalmente no incluye la tercera etapa del análisis de proporcionalidad. Id., pp. 416-431.

10 Alexander Aleinikoff, Constitutional Law in the Age of Balancing, 96 Yale Law Journal 943, 1987: 948-963.

11 VICKI JACKSON, Constitutional Law in the Age of Proportionality, 124 Yale Law Journal 3094 (2015), explica que, aunque muchas áreas del derecho constitutional estadounidense se rigen por la idea de la proporcionalidad, la jurisprudencia no ha producido una doctrina estructurada al respecto. Entre los factores que para la autora pueden explicar esta situación, conviene resaltar tres: a) a diferencia de muchas de las cortes europeas, las cortes estadounidenses se han mostrado reacias a elaborar una teoría de la constitución que conecte las diferentes cláusulas del texto constitucional de forma sistemática; b) por su antigüedad, la constitución de los Estados Unidos contiene menos derechos que la mayoría de constituciones modernas, lo que explica que los conflictos entre valores constitucionales sean menos frecuentes; y c) los Estados Unidos se encuentran relativamente aislados de las fuentes del derecho internacional de los derechos humanos, las cuales han contribuido a la expansión de la doctrina del principio de proporcionalidad en todo el mundo. Id., pp. 3121-29.

12 Mattias Kumm, The Idea of Socratic Contestation and the Right to Justification: The Point of Rights-Based Proportionality Review, 4 Law and Ethics Human Rights 141 (2010); Moshe CoHen-Eliya e Iddo Porat, Proportionality and the Culture of Justification, 59 American Journal of Comparative Law 463 (2011). 
órganos legislativos democráticos, bajo ciertas condiciones especiales, derogar prima facie derechos constitucionales en aras de la garantía de intereses públicos. El legislador puede entonces alcanzar objetivos constitucionales que gozan de apoyo popular. Además, si las cortes adelantan un juicio de proporcionalidad deferente con la legitimidad democrática del legislativo, y al analizar la idoneidad, necesidad y proporcionalidad de la medida en estricto sentido llegan a la conclusión de que esta no es simplemente "equivocada", sino que en realidad es "irrazonable", la tensión entre el control jurisdiccional y la democracia es atenuada de esta forma ${ }^{13}$.

Como veremos a continuación, las tres preguntas que constituyen la base del principio de proporcionalidad solo pueden ser formuladas y contestadas sobre la base de una teoría sustantiva de la justicia y los derechos. Las difíciles cuestiones normativas que los jueces deben abordar al resolver casos particulares son de alguna forma "externas" a los mecanismos del principio de proporcionalidad ${ }^{14}$. En este capítulo, deseo concentrarme en ciertos asuntos que ilustran esta necesidad de una teoría sustantiva.

\section{DELIMITAR DERECHOS ABSOLUTOS Y DERECHOS RELATIVOS}

Una primera idea que conviene subrayar es que el principio de proporcionalidad solo puede ser aplicado cuando el derecho en cuestión es susceptible de ser restringido. La mayoría de derechos lo son, por lo que son comúnmente llamados "derechos relativos". Otros derechos, sin embargo, son "absolutos", por cuanto la legitimidad de cualquier restricción a los mismos es denegada por completo. En estos casos, determinar si la restricción bajo examen puede considerarse legítima a la luz del test de proporcionalidad es una pregunta irrelevante, pues los derechos absolutos son inmunes a cualquier ejercicio de este tipo.

13 Stephen Gardbaum, A Democratic Defense of Constitutional Balancing, 4 Law and Ethics Human Rights 78 (2010).

14 Así ha sido reconocido por los mejores expertos en proporcionalidad. Barak, supra nota 2,480 , por ejemplo, afirma: "Proportionality is based on the reasons underlying each constitutional right, as well as the justifications for their limitation. These reasons and those justifications in and of themselves, however, are extrinsic to the concept of proportionality. Proportionality is unable to resolve those issues". De manera similar, Kai Möller, en The Global Model of Constitutional Rights (2012), insiste en que la proporcionalidad y la ponderación son herramientas doctrinarias que los jueces utilizan para examinar las cuestiones morales más importantes en la resolución de casos constitucionales. "The danger", afirma, "is that, if misunderstood, they may suggest a mistaken simplicity about reasoning with rights: the idea that the difficult questions about rights can be resolved by recourse to a largely mechanical exercise of quantification" Id. 207 . Sin embargo, David Beatty en The Ultimate Rule of Law 166-76 (2004), señala que la doctrina de proporcionalidad permite a los jueces reconducir cuestiones normativas a preguntas sobre la lógica o los hechos. 
Una forma de realizar esta distinción entre derechos absolutos y relativos consiste en afirmar que los primeros se encuentran previstos en normas constitucionales que revisten la estructura de "reglas", al tiempo que las normas que consagran derechos relativos revisten la estructura de "principios" Una regla expresa un lenguaje categórico, imperativo, mientras un principio contiene un lenguaje más flexible. Lo que establece una regla no es susceptible de excepciones implícitas que puedan justificarse con base en consideraciones externas. Por el contrario, un principio está abierto a este tipo de ponderación (balancing), pues exige que algo sea alcanzado en la mayor medida posible, teniendo en cuenta la existencia de otros principios y reglas que puedan resultar contradictorias ${ }^{16}$.

Es importante tener claro que todos los derechos, absolutos o relativos tienen un alcance limitado. Tenemos un derecho a algo (libertad de expresión, libertad de asociación, a no ser torturados, etc.), por lo cual el contenido del derecho requiere ser definido. Existe entonces un límite que distingue aquello que se encuentra dentro del ámbito de aplicación del derecho, y aquello que excede el alcance de este derecho. Sin importar cuán amplio sea el alcance de un derecho en particular, siempre existirá un límite más allá del cual el derecho no resulta aplicable. Será labor del intérprete determinar el alcance particular de la norma constitucional que consagra un derecho específico. El principio de proporcionalidad solo entrará en juego una vez el alcance del derecho haya sido determinado a través de la interpretación ${ }^{17}$. Sin embargo, como es natural jueces de diferentes jurisdicciones que interpretan distintos textos constitucionales en el marco de sus respectivas tradiciones jurídicas llegarán a conclusiones divergentes respecto del alcance de un mismo derecho constitucional. Es cierto que la estructura abierta y la formulación abstracta de las normas constitucionales facilita la convergencia, pero las diferencias interpretativas son inevitables ${ }^{18}$.

De esta forma, la distinción entre derechos absolutos y relativos nos conduce a preguntarnos si, una vez delimitado el alcance de un derecho, las acciones que se encuentren dentro del ámbito de aplicación del derecho siempre estarán protegidas por el mismo, sin excepciones, o si por el contrario estas acciones solo estarán protegidas bajo la condición de que su garantía no sea derrotada por otras consideraciones de mayor peso.

15 Ver Alexy, supra nota 5,pp. 47-48.

16 Conviene aclarar que el "principio" de proporcionalidad no es en realidad un principio, sino una regla. Lo que ordena (que las limitaciones de derechos deben ser proporcionales), lo ordena en forma categórica.

17 BARAK, supra nota 2, pp. 45-82.

18 Como afirma Grant Huscroft, "the decision to adopt a bill of rights is a decision to give heightened legal status to particular rights, rather than to rights in general". Ver Proportionality and the Relevance of Interpretation, en Proportionality and the Rule of Law 188 (Grant Huscroft, Bradley W. Miller y Grégoire Webber, eds., 2014). 
Necesitamos entonces una teoría que nos permita dilucidar cuáles derechos pertenecen a cada una de estas categorías. Sin embargo, el principio de proporcionalidad guarda silencio al respecto, y solo resulta aplicable una vez resuelta la cuestión anterior. Como veremos, determinar cuáles derechos son absolutos es una tarea más difícil de lo que parece.

Antes de avanzar en este sentido, debemos tener en cuenta que la idea de que las limitaciones a los derechos puedan estar justificadas en ocasiones lleva implícita la idea de que algunos conflictos pueden surgir entre la garantía de los derechos y otros valores constitucionales (otros derechos e intereses). Algunos académicos han enfatizado en la necesidad de interpretar de manera holística los distintos valores y principios que la constitución considera legítimos. Sería absurdo afirmar, por ejemplo, que si yo disfruto el hecho de asesinar otras personas y quisiera matar a alguien, existiría un conflicto entre mi derecho constitucional a la libertad y el derecho a la vida. En este caso, el alcance del derecho a la libertad debe interpretarse a la luz del derecho a la vida. Cuando la ley me prohibe matar, no está restringiendo mi libertad, por cuanto esta no abarca la acción de quitarle la vida a una persona ${ }^{19}$. Por lo anterior, este llamado a la interpretación armónica debe ser aplaudido. En realidad, muchos conflictos resultarán tan solo aparentes si interpretamos las normas constitucionales relevantes de forma correcta, si hacemos que estas encajen en un esquema coherente.

Sin embargo, sería erróneo considerar que ningún conflicto genuino podría permanecer tras un correcto ejercicio de interpretación. Nos podemos encontrar ante situaciones en las cuales un derecho debe ser sacrificado parcial o totalmente en aras de promover un objetivo legítimo de suficiente relevancia constitucional. Entonces, es cierto que mi derecho a la libertad no se ve "restringido" cuando la ley me prohibe matar, sin importar en qué medida esta prohibición disturbe mis planes. Sin embargo, si la ley establece una larga pena de prisión para mí como castigo por cometer homicidio, allí sí surge un conflicto entre mi derecho a la libertad y la política criminal que el Gobierno busca implementar mediante el castigo. La prisión es una privación de la libertad en todo caso, aunque sea una privación justificada. Para usar un ejemplo más extremo: si la pena de muerte estuviera justificada

19 Ver Ronald Dworkin, Do Liberal Values Conflict?, en The Legacy of Isaiah Berlin 88-89 (Mark Lilla, Ronald Dworkin y Robert B. Silvers eds., 2001). Kai Möller, sin embargo, considera que el principio de proporcionalidad exige una "inflación de derechos": todos los intereses autónomos deben ser protegidos como derechos, incluídos los intereses de involucrarse en actividades triviales o incluso inmorales. Según Möller, existe en realidad un "derecho a asesinar", aunque a su vez existen sin lugar a dudas razones de peso para restringir este derecho, en aras de proteger el derecho a la vida de los demás. Pero esto no significa, desde su perspectiva, que no podamos reconocer la existencia de un derecho a asesinar (por más sencillo de derrotar que resulte). Ver KAI Möller, Proportionality and Rights Inflation, en Proportionality and the Rule of Law, supra nota 18, pp. 155-172. 
en ciertas circunstancias especiales, sería difícil negar que el derecho a la vida del criminal está en juego. Incluso si la privación de libertad estuviera justificada, la pena sería una restricción a su derecho a la vida.

En realidad, nosotros como sociedad necesitamos establecer un sistema de justicia criminal en aras de alcanzar los objetivos retributivos y disuasivos del castigo, incluso si sabemos que existe el riesgo de tomar decisiones equivocadas. Debido a la escasez de recursos y las limitaciones temporales, y más importante aún, debido a la falibilidad humana, no podemos garantizar que ninguna persona inocente será condenada. Esperamos reducir este riesgo de equivocaciones en la mayor medida posible mediante las garantías procesales pertinentes, pero sabemos que los accidentes son inevitables. La maquinaria institucional nunca será perfecta. A pesar de todo, consideramos que el sistema de justicia criminal se justifica a la luz de las consecuencias negativas que traería consigo su eliminación por completo. Pero somos conscientes de la trágica decisión tomada. Cuando descubrimos que una persona inocente fue encarcelada erroneamente por la comisión de un delito, lo lamentamos profundamente, y con frecuencia creemos que esa persona merece una compensación de algún tipo. No obstante, sería absurdo negar la existencia de un conflicto subyacente entre libertad y la necesidad pública de establecer un sistema que brinde justicia y provea castigos ${ }^{20}$.

\section{A. Carácter absoluto e importancia del derecho}

Entonces: ¿cuáles derechos constitucionales son absolutos? Resulta tentador afirmar que son absolutos aquellos derechos constitucionales que protegen los intereses jurídicos más importantes en una sociedad liberal y democrática. Por el contrario, serán relativos aquellos derechos que protegen intereses de una importancia menor. Algunos ejemplos de derechos absolutos efectivamente se refieren a derechos vitales, tales como el derecho a no ser torturado, o el derecho a no ser víctima de esclavitud. Parece razonable afirmar que cuanto más importante resulte un derecho, más difícil resultará justificar restricciones a este, al punto que algunos derechos que revisten un carácter fundamental para los seres humanos deban ser considerados como absolutos.

Desafortunadamente, esta tarea es más complicada. Tomemos la constitución española como ejemplo que en su artículo 15 consagra que todos tenemos "derecho a la vida". Al mismo tiempo, en su artículo 17 establece que cualquier persona arrestada por la policía tiene derecho a ser llevada ante un juez competente, o ser liberada, dentro de las 72 horas siguientes al arresto. No existe duda sobre cuál derecho es el más importante de los dos,

20 De forma más general, una reflexión sobre las distintas instituciones y procedimientos utilizados socialmente para distribuir recursos extremadamente escasos puede encontrarse en Guido Calabresi y Philip Bobbit, Tragic Choices (1978). 
y por consiguiente cuál vulneración sería más gravosa. Si una persona tiene la oportunidad de elegir, razonablemente preferirá que el gobierno respete su derecho a no ser asesinada sobre su derecho a ser llevada ante un juez dentro de las 72 horas siguientes al arresto. No obstante, aunque el derecho a la vida es más importante que el otro derecho mencionado, es un derecho relativo. A pesar de su fundamental importancia, el derecho a la vida puede sacrificarse en ciertas circunstancias. Por ejemplo, es legítimo que la ley autorice asesinatos en ejercicio de la defensa legítima, siempre que concurran ciertas condiciones. Además, en aras de la defensa nacional, un gobierno puede estar legitimado para reclutar soldados para luchar una guerra, aunque ponga sus vidas en riesgo. En general, el gobierno permite a las personas el uso de automóviles, los cuales constituyen causan gran número de muertes cada año de conformidad con los reportes estadísticos. Por el contrario, en el derecho constitucional español no existe ninguna justificación legal para que la policía se excuse de su deber de llevar a todas las personas arrestadas ante los jueces en las 72 horas siguientes a su captura. Este último derecho es menor importante que el derecho a la vida, pero está protegido por la constitución de forma categórica e imperativa.

\section{B. Carácter absoluto y cláusula de intangibilidad}

La pregunta sobre el carácter absoluto o relativo de los derechos es independiente de la pregunta sobre si un derecho se encuentra incluido dentro de aquel "núcleo de derechos" que se encuentran protegidos frente a cualquier tipo de reforma constitucional. Como sabemos, un buen número de constituciones en el mundo establecen límites sustantivos al tipo de modificaciones que se pueden realizar mediante procesos de reforma constitucional. En ocasiones, un conjunto de instituciones, normas, principios, entre otros, son atribuidos un valor fundacional dentro del orden constitucional existente, al punto que se ubican más allá del alcance de los poderes de enmienda constitucional. Se han desarrollado distintas teorías para identificar aquellos derechos que deben ser incluidos (o ser tenidos en cuenta para ser incluidos) en este núcleo ${ }^{21}$. Sin embargo, existe cierto consenso respecto de que la democracia y los derechos de participación política que se derivan de ella pertenecen a este núcleo intocable cuando las constituciones prevén tal núcleo. El derecho al voto se entiende entonces protegido frente al efecto de las reformas constitucionales.

Sin embargo, lo anterior no significa que el derecho al voto sea necesariamente un derecho absoluto. Pueden existir razones de peso que justifiquen

21 Una discusión iluminadora de la doctrina de las reformas constitucionales inconstitucionales puede verse en Rosalind Dixon y David Landau, Transnational Constitutionalism and a Limited Doctrine of Unconstitutional Constitutional Amendments, 13 International Journal of Constitutional Law 606 (2015). 
limitaciones a este derecho. Si asumimos en aras de la simplicidad que los titulares de este derecho al voto son tan solo los ciudadanos mayores de edad, de tal forma que técnicamente no existe una "restricción" cuando la ley niega el voto a los extranjeros y los menores de edad, incluso allí es posible que la ley limite el ejercicio de este derecho en ciertos casos. Por ejemplo, existe controversia sobre la posibilidad de suspender el ejercicio del derecho al voto de las personas culpables de delitos graves, durante el tiempo en que cumplen sus condenas. Aunque esta controversia excede el objeto del presente texto, es importante resaltar el hecho de que no es conceptualmente erróneo afirmar que, aunque el derecho al voto sea parte de aquel núcleo protegido frente a procesos de reforma constitucional, es legítimo que la ley restrinja el ejercicio de este derecho a las personas que han cometido graves delitos.

\section{Carácter absoluto y suspensión de derechos}

La cuestión del carácter absoluto de un derecho también debe distinguirse del problema relativo a la posibilidad de que un gobierno "suspenda" la garantía de un derecho en casos de emergencia. En muchas jurisdicciones, es posible suspender o derogar ciertos derechos bajo circunstancias excepcionales. Sin embargo, no todos los derechos son susceptibles de ser derogados o suspendidos. Algunos derechos están blindados frente a este tipo de medidas. Una vez más, los derechos absolutos no corresponden a aquellos derechos que no pueden ser suspendidos en situaciones de emergencia.

La constitución española resulta útil para ilustrar este punto. Esta constitución permite al Gobierno la suspensión de ciertos derechos cuando sea decretado un estado de sitio o excepción ${ }^{22}$. Como vimos anteriormente, el derecho de una persona arrestada a ser llevada ante los jueces dentro de las 72 horas siguientes a su captura es un derecho absoluto. Sin embargo, este derecho puede ser suspendido en situaciones de emergencia. Por el contrario, el derecho a la vida, que no es un derecho de naturaleza absoluta, está blindado frente a este tipo de suspensiones en caso de emergencia. De esta forma, no existe coincidencia entre el conjunto de derechos absolutos y el conjunto de derechos que no pueden ser suspendidos cuando el estado de sitio o excepción sea declarado.

\section{Identificar derechos absolutos: las limitaciones del lenguaje constitucional}

Ahora, una vez aclarado el panorama conceptual tras haber distinguido los derechos absolutos de aquellos derechos que revisten otras características normativas e institucionales, la tarea difícil debe llevarse a cabo: debemos 
determinar cuáles derechos son absolutos (aquellos contenidos en reglas categóricas), y cuáles son relativos (aquellos contenidos en principios que permiten un ejercicio de ponderación).

El lenguaje de la constitución será entonces un punto de partida para este propósito. Supongamos que los redactores de la constitución eran plenamente conscientes de la necesidad de diferenciar derechos absolutos de aquellos relativos. Si el texto constitucional escrito por ellos enlistara explícitamente aquellos derechos que no permiten ejercicios de ponderación, pareciera entonces que no existe la necesidad de construir una teoría sustantiva en este sentido. Sin embargo, la realidad es que por regla general no existe tal lista de derechos en los textos constitucionales. Debemos entonces analizar cada norma particular, en aras de determinar si esa cláusula particular se expresa o no en forma categórica o imperativa.

Resulta interesante el hecho de que cuando una norma constitucional incluya tanto la regla como su excepción explícita, esto parezca indicar que la norma se expresa en forma categórica. Precisamente, porque se establece una excepción explícita podemos asumir que los redactores de la constitución pensaron en las posibles excepciones a este derecho y concluyeron que tan solo las excepciones particular que estipularon se encuentran justificadas. Lo mismo sucede cuando más de una excepción es estipulada expresamente. Una vez se encuentra determinado el alcance del derecho mediante la regla y las excepciones pertinentes, pareciera que ninguna restricción del derecho se encuentra permitida. El juez probablemente no podrá emplear el principio de proporcionalidad para justificar una excepción adicional a la regla que consagra el derecho.

La constitución española, por ejemplo, establece en su artículo 15 "Queda abolida la pena de muerte, salvo lo que puedan disponer las leyes penales militares para tiempos de guerra". El lenguaje utilizado reviste tal expresión categórica, que resulta difícil afirmar que el derecho a no ser condenado a pena de muerte pueda ser restringido (aparte de las excepciones referidas a tiempos de guerra). Sin importar los intereses imperiosos que el gobierno pueda invocar para justificar la pena de muerte, estos resultan absolutamente irrelevantes por cuanto no podrán derrotar la regla constitucional que consagra la abolición de esta pena capital, una regla cuya única excepción es una situación de guerra.

Sin embargo, también podría afirmarse que no tiene sentido clasificar como "absolutos" aquellos derechos previstos en normas que establecen al tiempo la regla y su excepción. Si la constitución explicitamente afirma que la pena de muerte puede ser impuesta en tiempo de guerra, por ejemplo, entonces el derecho a no ser castigado con la pena de muerte no es absoluto. Lo que la constitución establece es categórico, pero el derecho no es absoluto toda vez que la regla estipulada una excepción explícita a la garantía del derecho. Este es un punto válido y podríamos simplemente distinguir entre reglas y 
principios - esto es, entre normas categóricas y normas abiertas a la posibilidad de ponderación - y descartar la distinción entre derechos absolutos y relativos. Sin embargo, no existe ningún inconveniente en recurrir a esta última distinción si somos cuidadosos y la usamos para referirnos al contraste entre reglas categóricas y principios flexibles ${ }^{23}$.

No obstante, en ocasiones los argumentos que se basan en la estructura de regla y excepción de una determinada norma constitucional para afirmar que un derecho es protegido de manera categórica son derrotados. La constitución española contiene un buen ejemplo de esta situación. En su artículo 18.2 proclama el derecho a la inviolabilidad del domicilio de las personas. En particular, establece que "Ninguna entrada o registro podrá hacerse en él sin consentimiento del titular o resolución judicial, salvo en caso de flagrante delito". Esto parece expresar un lenguaje bastante categórico. Entonces, ¿significa esto que un bombero requiere una orden judicial para entrar a una casa y extinguir un incendio? Por supuesto que no. El Tribunal Constitucional estableció que en relación con este derecho previsto en el artículo 18.2 existe una excepción implícita: la entrada al domicilio se encuentra autorizada si una situación de "estado de necesidad" tiene lugar ${ }^{24}$. Considerar que este derecho (tal y como se encuentra consagrado por la regla constitucional con dos excepciones implícitas) es absoluto podría traer consigo resultados difíciles de justificar desde un punto de vista normativo.

En la práctica, debemos reconocer que no tiene mucha relevancia la forma en que califiquemos las excepciones implícitas que establece el texto constitucional al consagrar un derecho en particular. Podemos considerar que estas excepciones hacen parte de la definición del mismo derecho (como calificativos internos que determinan el alcance del derecho), o podemos afirmar que se trata de restricciones que los redactores de la constitución decidieron imponer - o permitieron que el legislador impusiera - a este derecho. En ocasiones, una de estas dos interpretaciones resulta más "natural" que la otra. Por ejemplo, podríamos afirmar que el requisito de que una reunión sea "pacífica" para gozar de protección constitucional hace parte de la definición interna del derecho de reunión o asociación, mientras

23 El concepto de derechos absolutos es más problemático de lo que parece. Ver AlaN GewiRTh, Are There Any Absolute Rights?, 31 Philadelphia Quarterly 1 (1981). Tal y como lo expone el autor, el problema es el siguiente: "Since an absolute right is one that is valid without exceptions, it may be concluded either that no rights are absolute because all involve some specification, or that all rights are equally absolute because once their specifications are admitted they are entirely valid without any further exceptions". Id. 5. Una forma de solventar esta dificultad es entender que ciertas especificaciones no impiden que el derecho sea absoluto. Según Gewirth, si el derecho no está sobrecargado de excepciones, si las especificaciones se encuentran justificadas mediante un principio moral válido, y si cualquier referencia a las posibles consecuencias negativas de satisfacer el derecho es excluida, entonces el derecho puede aún ser considerado absoluto, a pesar de aquellas especificaciones.

24 sTC 22/1984, de 17 de febrero. 
la cláusula que establece que la pena de muerte puede ser impuesta durante situaciones de guerra debe ser entendida como una restricción externa al derecho a no ser castigado con pena de muerte. Para efectos del presente texto, sin embargo, dejaremos a un lado esta diferencia. La cuestión central aquí es la siguiente: una vez el intérprete ha definido el alcance del derecho teniendo en cuenta las excepciones explícitas que el texto constitucional establece (sean estas consideradas parte de la definición interna del derecho, o restricciones externas al mismo), ¿puede el Gobierno establecer limitaciones adicional al derecho con fundamento en razones que no están cobijadas por las excepciones explícitas?

Cuando las disposiciones constitucionales que garantizan derechos no mencionan excepción explícita alguna, resulta aún más difícil recurrir al lenguaje del texto para determinar si el derecho en cuestión es o no absoluto. La constitución puede proclamar que todas las personas tienen derecho a la "libertad de expresión". También puede establecer que cuando una persona es acusada de haber cometido un delito, será titular de una "presunción de inocencia" y el derecho a ser juzgada por un "tribunal imparcial". En ambos casos, el lenguaje parece similar. No obstante, parece razonable afirmar que el derecho a la libertad de expresión no es absoluto - otros derechos o intereses, como la privacidad o la seguridad nacional, por ejemplo, pueden limitarlo- mientras el derecho a la presunción de inocencia y a un tribunal imparcial sí es absoluto: ningún juez puede declarar la culpabilidad de una persona acusada de la comisión de un delito sin la existencia de pruebas suficientes que acrediten esta declaración de conformidad con el razonamiento judicial del caso; y ningún proceso judicial puede considerarse válido si el tribunal que lo ha adelantado no es imparcial ${ }^{25}$.

Conviene dejar claro aquí que la definición del derecho a la presunción de inocencia, al igual que el derecho a un tribunal imparcial, está relacionado con ciertas características que son susceptibles de graduación. El estándar probatorio requerido para rebatir la presunción de inocencia, por ejemplo, puede ser más o menos demandante. ¿Cuán fuerte debe ser la evidencia que indica la culpabilidad del acusado? En aras de definir el estándar probatorio, el legislador ha debido ponderar diferentes consideraciones normativas. Entre otras cosas, ha debido valorar la relativa importancia normativa de dos tipos diferentes de errores que los jueces (o los jurados) pueden cometer: inculpar al inocente, o absolver al culpable. El estándar será más estricto si el legislador se enfoca en evitar en lo posible la encarcelación de personas inocentes. Sin embargo, una vez establecido, el estándar hace parte del derecho. Debido a la naturaleza absoluta del derecho, los jueces no pueden desconocer el

25 Dejo a un lado la pregunta sobre si es contrario a la presunción de inocencia el hecho de que la ley le exija a la persona acusada de la comisión del delito que pruebe los fundamentos de su defensa o refutar presunciones legales. 
estándar probatorio que el derecho supone en nombre de intereses sociales contrapuestos. De manera similar, la imparcialidad de los jueces depende de ciertas características que son susceptibles de graduación, tales como la "distancia" existente entre el juez y las partes. Sin embargo, existe un límite que debe satisfacer el juez para ser considerado imparcial. Una vez el derecho a un tribunal imparcial ha sido definido de esta manera, el derecho no puede ser restringido en nombre de consideraciones enfrentadas.

Como hemos visto, el lenguaje del texto constitucional no es de mayor ayuda a la hora de establecer si un derecho es o no susceptible de ponderación. Debemos construir una teoría sustantiva para cumplir este propósito. Debemos pensar en cada derecho y descubrir, mediante la mejor interpretación posible del derecho a la luz de sus fundamentos normativos, si existen o no razones que justifiquen posibles restricciones del derecho. Algunos derechos son de tal naturaleza que al interpretarlos, cualquier objetivo constitucional debe ser excluido como fuente legítima de limitaciones al derecho.

\section{IDENTIFICACIÓN DE FINES LEGÍTIMOS}

La implementación del principio de proporcionalidad requiere que los jueces especifiquen los fines legítimos que justifican limitaciones de derechos, teniendo en cuenta su naturaleza relativa y de este modo, abiertos a la ponderación.

La primera pregunta que surge en esta relación es si los derechos fundamentales garantizados en la Constitución solo se pueden restringir en el nombre de otro derecho fundamental. La Declaración Francesa de Derechos Humanos de 1789 consagra en su artículo $4^{\circ}$ que "el ejercicio de los derechos naturales de cada hombre tan sólo tiene como límites los que garantizan a los demás Miembros de la Sociedad el goce de estos mismos derechos". Esta interpretación de los derechos, sin embargo, corresponde en nuestros días a una visión demasiado estricta. El interés público también puede ser una fuente legítima de restricciones. La Declaración Universal de Derechos Humanos de 1948 marca la pauta generalizada, cuando establece lo siguiente en el artículo 29 (2):

En el ejercicio de sus derechos y en el disfrute de sus libertades, toda persona estará solamente sujeta a las limitaciones establecidas por la ley con el único fin de asegurar el reconocimiento y el respeto de los derechos y libertades de los demás, y de satisfacer las justas exigencias de la moral, del orden público y del bienestar general en una sociedad democrática.

Generalmente se acepta que existan interferencias en el ejercicio de un derecho fundamental, no solo con el fin de proteger otro derecho que también tiene una categoría relevante dentro de la Carta, sino también para proteger otro derecho que carece de este status, o para proteger el interés público, siempre 
y cuando estos otros derechos e intereses posean suficiente importancia ${ }^{26}$. Así pues, los derechos no se consideran como "triunfos" que siempre prevalecen sobre el interés público (salvo que acaezca una verdadera situación catastrófica). Resulta más adecuado concebirlos como "escudos", si utilizamos la metáfora de Frederick Schauer. Los derechos fundamentales, en general, protegen a los individuos contra las restricciones que se adoptan en nombre del interés público, pero no los protegen contra aquellas restricciones que son necesarias para preservar intereses públicos de suficiente importancia ${ }^{27}$.

En este orden de ideas, es posible sostener, por ejemplo, que la libertad de expresión puede ser limitada, bajo condiciones adecuadas, si la información que se pretende difundir pone en serio riesgo la seguridad nacional. Este último bien es un interés público importante, que justifica las restricciones a la libertad de expresión, incluso cuando no existe un "derecho fundamental a la seguridad nacional".

Es posible, por supuesto, desagregar los componentes del interés público (seguridad nacional, orden público, etc) y asegurar que, en últimas, estos consisten en intereses particulares protegidos por los derechos fundamentales. La seguridad nacional, por ejemplo, tiene un vínculo instrumental con la protección de la vida de los ciudadanos contra ataques externos. En otras palabras, el derecho a la vida es la base o sustento de la seguridad nacional. Si pensamos bajo esta lógica, borraremos la distinción entre derechos e interés público, y así, la tesis de que los derechos fundamentales solo pueden restringirse en nombre de otros derechos de la misma categoría pierde su carácter original.

Partiendo de la base de que los fines que pueden justificar la limitación de derechos fundamentales no hacen referencia exclusiva a otros derechos fundamentales, es válido preguntar cuáles son los fines legítimos para tales limitaciones. A menudo, los textos de las Constituciones o las convenciones internacionales más relevantes consagran estos fines relevantes. De vez en cuando, en ellas se incluye una cláusula general, que aplica a todos los derechos que están consagrados en el texto ${ }^{28}$. Es más frecuente, por el contrario, que el artículo que consagra el derecho especifique el fin que puede invocarse con el fin de justificar la imposición de determinada restricción sobre el mismo ${ }^{29}$.

$26 C f r$. BARAK, supra nota 2,pp. 260-285 para un estudio comparado de esta problemática.

27 Frederick Schauer, A Comment on the Structure of Rights, 27 Ga L. Rev. 415 (1993).

28 Cfr. A.G Res 217 (III) A, Declaración Universal de los Derechos Humanos, art. 29 párr. 2 (10 de diciembre de 1948); Canadian Charter of Rights and Freedoms, Part I of the Constitution Act, 1982, being Schedule B to the Canada Act, c 1 (U.K.); S. Afr. Const., ch. 2 36; Human Dignity and Liberty, 5752-1992, par 8. (1992) (Isr).

29 Esta es la estrategia que adopta el Convenio Europeo de Derechos Humanos. También es la posición dominante a nivel nacional. Como apunta Aharon Barak, "el método prevalente para limitar derechos constitucionales en las constituciones modernas es a través de la adopción de algunas cláusulas de limitación”. BARAK, supra note 2, at 141. 
Es posible argumentar a favor de un enfoque diferenciador. Un propósito particular puede ser legítimo en abstracto, pero esta legitimidad puede estar sujeta a restricciones que aplican a determinados derechos, y no a otros.

Tomemos como ejemplo el interés del estado en promover valores de una democracia liberal. Supongamos que aceptamos que, si tales valores no están generalizados de manera amplia en la sociedad, las instituciones de la democracia liberal no pueden funcionar correctamente y pueden colapsar. En abstracto, inculcar estos valores en los ciudadanos es un fin legítimo para el gobierno. Esto no significa, sin embargo, que todos los derechos puedan restringirse para conseguir dicho fin. Por ejemplo, el gobierno puede limitar el derecho de los padres a educar a sus hijos de acuerdo con sus creencias religiosas y morales en el contexto del sistema de educación, con el fin de promover ciertos valores públicos dentro de la población más joven. Por el contrario, no es posible partir de la misma base para limitar la libertad de expresión. La protección de este derecho debe extenderse incluso a aquellos discursos que atacan los valores de la democracia liberal.

Es importante resaltar, para la elaboración de esta distinción, que no necesitamos afirmar que la libertad de expresión es más importante que el derecho de los padres a elegir la educación de sus hijos. Lo que necesitamos demostrar es que la libertad de expresión excluye un objetivo específico como suficientemente relevante para justificar una restricción a este derecho, mientras que el derecho de los padres no. En otras palabras, parte de lo que significa que se conceda un derecho particular es que se excluyen ciertas razones potenciales para restringir ese derecho. Estas razones pueden ser legítimas en abstracto, y pueden funcionar desde un plano normativo cuando otros derechos están en juego. Si esta idea es plausible, estamos entonces obligados a desarrollar una teoría que identifique qué tipo de fines deben ser excluidos en la etapa de justificación, para cada derecho ${ }^{30}$.

Cuando pensamos en los objetivos que podrían servir como fundamento para limitar algún derecho, es importante ser conscientes de la teoría que subyace a la protección de cada derecho. Ya se ha mencionado, por ejemplo, la controversia sobre si debe haber una privación del derecho al voto como parte de una pena por haber cometido determinados delitos. En abstracto, podría decirse que la privación del derecho al voto está justificada en la disuasión y la retribución. Sin embargo, este argumento es muy abstracto. Antes de analizar la legitimidad de la medida, es necesario pensar de manera más profunda sobre los fundamentos del derecho al voto.

Si entendemos que el derecho al voto está garantizado para permitir a cada persona defender sus intereses en medio del proceso político, será difícil justificar la restricción: las personas que se encuentran cumpliendo una condena

30 Para una aproximación similar, cfr. RiCHARD H. PILDERs, Avoiding balancing: the role of exclusionary reasons in constitutional law, 45 Hastings L.J. 711 (1994). 
penal en una cárcel tienen intereses políticos importantes por proteger. Por ejemplo, un cambio en la legislación podría reducir el tiempo de la pena. Si en vez de esto, el derecho al voto está vinculado con una concepción diferente de democracia, de acuerdo con la cual él o la votante expresan su visión sobre el interés general, bajo la lógica de los derechos fundamentales de todas las personas, se abren más posibilidades para justificar restricciones a dicho derecho. Esta es una cuestión compleja, y muchas consideraciones pueden ir en direcciones opuestas. Sin embargo, es necesario tener alguna idea acerca de cuál es el núcleo del derecho al voto antes de evaluar cuan proporcional puede ser su restricción, en el caso de personas que han cometido delitos.

Una vez se acepta que un fin es legítimo de cara a restringir un derecho determinado, aún hay un arduo trabajo normativo por hacer, en aras de especificar su contenido en contextos particulares. Piénsese, por ejemplo, en las controversias respecto de la forma en que aparecemos en público. ¿Podría el gobierno solicitar que no se cubran los rostros, o que cubramos parte de nuestro cuerpo para que no aparezcamos desnudos?

La Corte Europea de Derechos Humanos ha decidido este tipo de casos. Por ejemplo, en el caso S.A.S v. Francia del 2014, el Estado profirió una ley que prohibió cubrir la cara en público, bajo el argumento de que la restricción era necesaria para proteger los derechos de los demás miembros de la comunidad. Tanto el gobierno francés, como la Corte, sostuvieron que los miembros de la sociedad tienen derecho a vivir en un espacio de socialización que les permita materializar el principio de "vivre ensemble" ${ }^{31}$. Asimismo, en el caso Gough v. Reino Unido del mismo año, la Corte convalidó una ley que prohibía la desnudez en las calles, bajo el entendido de que los demás tenían derecho a no ser perturbados por conductas antisociales y ofensivas ${ }^{32}$.

Tanto estos como otros casos resultan controversiales. Ellos ilustran la necesidad de ahondar en la teoría normativa sustantiva para especificar las condiciones que delimiten el alcance de los "derechos de los demás" para dar fundamento a las limitaciones del derecho de algún individuo a aparecer como desee en sitios públicos. No es plausible que la decisión se fundamente en el número de personas que se sienten ofendidas, ni en la intensidad de este sentimiento. Estaríamos vulnerando los derechos individuales de una manera apresurada si aceptáramos como una razón suficiente para que el Estado prohíba una forma de apariencia en particular el hecho de que la mayoría de los ciudadanos se encuentran profundamente ofendidos por esta. Es necesario una mejor teoría, sin embargo, ¿cómo se puede construir? ¿Cuáles son las perturbaciones que el derecho está llamado a evitar? ¿Debería ser relevante que andar desnudo o con la cara cubierta se hiciera a modo de protesta?

31 Caso S.A.S v. Francia, 2014. Tribunal Europeo de Derechos Humanos, 695.

32 Caso Gough v. Reino Unido, 2014. Tribunal Europeo de Derechos Humanos, 2014, 1156 . 
Preguntas de este talante solo se pueden responder con base en una teoría sustancial debidamente articulada.

PONDERACIÓN (BALANCING) Y EL PROBLEMA DE LOS PESOS ABSTRACTOS

Como se explicó con anterioridad, la fórmula tripartita del principio de proporcionalidad consiste en instruir a los jueces para que determinen si la limitación que analizan (i) es adecuada (o al menos en parte) para alcanzar un fin legítimo; (ii) necesaria, es decir, que no existe una alternativa más adecuada para conseguir dicho fin; y (iii) proporcional.

Es importante resaltar que el conflicto entre el derecho que se restringe, por un lado, y el derecho o interés que se defiende, por el otro, solo surge si la limitación resulta adecuada y necesaria. Es obvio que, si la limitación no es adecuada ni necesaria, no hay necesidad de limitar el derecho. Solo cuando los elementos de necesidad y adecuación concurren podemos hablar del surgimiento de un conflicto que obliga a elegir entre una u otra opción. En este momento es cuando hay lugar a la ponderación. En este punto la ponderación entra en escena.

En muchos países, el test de proporcionalidad en sentido estricto tiene gran relevancia, mientras que los otros dos pasos del test son aplicados por los jueces de forma bastante deferente. Muchos jueces entienden que para que una medida sea considerada idónea o adecuada, no resulta necesario que exista certeza de que esta medida promoverá directamente el objetivo perseguido. Tan solo un grado de probabilidad es suficiente. Sin embargo, la medida será necesaria incluso si existen otras medidas menos restrictivas para el derecho: si la última medida resulta más costosa, o resulta menos efectiva para alcanzar el fin perseguido, la primera medida seguirá siendo considerada como necesaria.

Así las cosas, el tercer estadio del test de proporcionalidad tiende a ser fundamental. En esta etapa, los jueces deben comparar, por una parte, el grado de la restricción del derecho contemplado en la ley o la decisión objeto de análisis, y por otra, el grado de satisfacción del fin perseguido. Tanto la intensidad de la restricción, como la contribución que hace la ley, o la decisión judicial a la realización del fin perseguido, variarán según el caso concreto. Según Robert Alexy, los jueces deben aplicar la teoría de la ponderación, según la cual "la limitación de un derecho constitucional solo es admisible si los principios que se contraponen al derecho subyacente tienen mayor peso en las circunstancias del caso" ${ }^{33}$. El contexto importa: cuando un juez revisa 
una medida que restringe un derecho, debe evaluar si las restricciones que acarrea pueden compensarse con los beneficios que se obtendrían con esta ${ }^{34}$.

En esta etapa, los números pueden resultar relevantes: con el fin de determinar la intensidad de una determinada limitación, las cortes pueden tener en cuenta la cantidad de personas que se verán afectadas, así como la cantidad de personas que se verán beneficiadas con la materialización del fin determinado.

El ejercicio de ponderación es, entonces, contextual. Sin embargo, el contexto es solo una parte de la historia. Los jueces necesitan determinar, además, qué tan relevantes son los derechos e intereses en comparación con el peso abstracto. Por supuesto, no hay una máquina que permita calcular el peso de los diversos derechos e intereses. Los jueces deben acudir a su intuición. Sin embargo, el peso abstracto es importante ${ }^{35}$. De acuerdo a Aharon Barak, "debemos distinguir entre status constitucional y el peso social". La importancia social de los derechos de orden constitucional puede variar ${ }^{36}$.

Por ejemplo, el Gobierno podría decidir, de manera acertada, que una manifestación no se puede realizar en el lugar elegido por los organizadores inicialmente, sino que debe realizarse en otra locación, toda vez que hay riesgo de que las ambulancias no puedan llegar a tiempo a un hospital, dado el alto flujo vehicular. Incluso si se restringe el derecho de reunión de miles de personas, y si no hay certeza (solo un grado suficiente de seria amenaza) de que una persona va a morir si la ambulancia tiene dificultades para llegar a tiempo al hospital, podríamos aceptar la restricción. Podríamos confiar en la intuición de que el derecho a la vida tiene más peso en abstracto que el derecho de asociación.

La aplicación del principio de proporcionalidad requiere de una teoría que articule este tipo de juicios intuitivos con los pesos abstractos. Como sostiene Frederick Schauer, requerimos "reglas del peso" para aplicar el principio

34 Barak presenta una versión más sofisticada de la teoría de la ponderación, supra nota 2, pp. 350-358. De acuerdo con este test, los jueces necesitan analizar la importancia social del margen de beneficio que se obtendría a través del cumplimiento de un propósito a través de una ley, y el margen de importancia social de prevenir la vulneración de un derecho constitucional causado por la ley. Cuando realice este ejercicio de ponderación, el juez deberá comparar la restricción con la situación legal que existía antes de que la medida se adoptara. El juez también debe comparar la medida con las demás medidas alternativas disponibles.

35 Alexy incluye los pesos abstractos en la "fórmula del peso" que propone, pero piensa que dichos pesos generalmente son equivalentes en casos de colisión de derechos constitucionales. Cfr. Robert Alexy, Formal Principles: Some Replies to Critics, 12 International Journal of Constitutional Law 511, 513 (2014).

36 BARAK, supra nota 2, p. 359. Barak propone dos niveles en la jerarquía de los derechos constitucionales, que deben integrarse en cada sistema jurídico nacional de acuerdo con las experiencias históricas particulares. Id, pp 531-532. Lo anterior puede resultar muy rígido. En un sistema dualista, es difícil lograr la coincidencia de la variedad de los pesos abstractos que se deben reconocer. 
de proporcionalidad, y allí pueden existir variaciones dependiendo de los distintos derechos en cuestión ${ }^{37}$.

En particular, si una Constitución incluye - o se incluye a través de su interpretación - un derecho general a la libertad, que implique que cualquier medida que suponga restricciones a los individuos deba ser llevada ante los jueces, o un derecho general a la igualdad, de modo que cualquier trato diferenciado, sin importar su naturaleza, pueda ser discutido ante los jueces, parecería posible que el juez constitucional pueda discriminar las distintas dimensiones de la libertad y la igualdad, y otorgarles pesos diferentes. Una cultura de la justificación requiere que el Estado justifique ante las cortes todas sus acciones en relación con los individuos, sin importar cuan triviales puedan resultar los intereses en juego. Según Mattias Kumm, en la práctica actual de varias cortes en el mundo, "las acciones para la protección de los derechos no se refieren de manera exclusiva a intereses considerados fundamentales, sino además a los mundanos" ${ }^{\prime 3}$. Sin embargo, la amplitud del ámbito constitucional hace necesario un análisis diferenciado. Las Cortes deben prestar atención a las diferencias cualitativas entre los derechos, así como a las diferencias cualitativas entre los diferentes componentes o ingredientes de los derechos, cuando involucren el ejercicio de la ponderación.

\section{ESTABLECER DIFERENCIAS CUALITATIVAS DENTRO}

DEL CAMPO DE OPERACIÓN DE UN DERECHO

Podríamos pensar que las dificultades que se han mencionado hasta el momento, respecto de los pesos abstractos, desaparecerían en aquellos casos donde el derecho que se invoca para justificar una limitación es precisamente el mismo que se está limitando. En estas circunstancias, no sería necesario comparar los pesos abstractos, siempre que el derecho que se encuentre en un lado de la balanza sea el mismo derecho que encontramos en el lado contrario. Pareciera que los jueces solo tuvieran que valorar el grado de afectación de cada derecho y el número de personas afectadas en cada caso.

Sin embargo, la realidad es más compleja. Por ejemplo, la ley permite matar a una persona en legítima defensa para salvar la vida de otra, bajo ciertas condiciones. El derecho a la vida de un agresor colisiona con el derecho a la

37 Frederick Schauer, Proportionality and the Question of Weight, in Proportionality and the Rule of Law, supra note 18, pp. 173-185. En el mismo sentido, $c f r$, Matthias KLatT y Moritz Meister, The Constitutional Structure of Proportionality 26, 29-42 (2012), sostienen que no se debe dar el mismo peso abstracto a todos los derechos constitucionales. Se debe dar mayor peso a derechos de vital importancia, como la vida y la dignidad.

38 Kumm, supra nota 12, p. 151. De igual forma, MöLleR, supra nota 14, p. 87, resalta que, de manera general, la práctica constitucional se ha movido hacia la "inflación de derechos", lo cual ha permitido la expansión de un espacio donde los gobiernos enfrenten la carga constitucional de justificar sus decisiones. 
vida de la víctima, y el derecho permite a la última (o a otras personas que la puedan ayudar) a sacrificar la vida del primero. De hecho, si existieran tres agresores, y la única manera de salvar la vida de la víctima es matarlos a todos, el derecho lo permite. El derecho distingue entre las vidas que están en juego. Los agresores han creado una situación de conflicto, que hace que su derecho a la vida ceda ante el derecho a la vida de la víctima. En este caso, el derecho presenta una distinción cualitativa, que triunfa sobre las consideraciones basadas en los números. En este punto, el derecho confía en un juicio de valore externo al principio de proporcionalidad.

Además, en ocasiones las diferentes dimensiones del mismo derecho están en conflicto. Muchos derechos son derechos de libertad, que presentan una dimensión positiva (el derecho a hacer $\mathrm{X}$ ), y una dimensión negativa (el derecho a no hacer X). El derecho a la libertad religiosa, por ejemplo, recoge el derecho a practicar una religión, así como el derecho a no practicarla. En algunos contextos, hay lugar a conflictos entre el derecho de una persona (o de un grupo) a ejercer la dimensión positiva de una libertad y el derecho de otra persona (o grupo) a ejercer la misma libertad en sentido negativo. La pregunta en este caso es si existe una buena razón para privilegiar un aspecto de un derecho sobre otro, cuando los dos colisionan.

Imaginemos, por ejemplo, un grupo de estudiantes en una escuela pública que quieren orar al inicio de la clase, mientras que otro grupo no quiere. Supongamos que hay un conflicto, en el que aquellos que no quieren orar se siente presionados, toda vez que se permitió la oración. Se puede proponer un argumento que da mayor peso al contenido adicional a la dimensión negativa: ser obligado a orar en la escuela, cuando no se quiere, es peor que no permitir orar cuando se desea. La razón de la asimetría tiene que ver en parte con la reversibilidad. Probablemente, el grupo que está siendo forzado a orar no puede hacer nada a futuro para compensar las consecuencias de dicha imposición, mientras que el grupo que al que no se le permite orar puede hacerlo después, fuera de la escuela, disminuyendo así el impacto de la prohibición.

En cierta medida, por supuesto, los números importan, pero en este punto parece haber una distinción cualitativa, entre el componente positivo y negativo de la libertad religiosa, que puede ser relevante. ¿Este tipo de argumento es convincente?

De igual forma, se ha argumentado que el motivo para prohibir el uso del velo en público se basa en que algunas mujeres musulmanas son obligadas a usarlo por sus familias. ¿Debemos distinguir entre la dimensión positiva y negativa de la libertad religiosa, y dar más peso a la última?

Un ejemplo dramático de tensión se presenta en contextos de controversia sobre el suicidio asistido de personas con enfermedades terminales. Por ejemplo, en el caso Pretty v. Reino Unido de 2002, el Tribunal Europeo de Derechos Humanos revisó una solicitud de una mujer que padecía una 
enfermedad incurable y degenerativa, que aducía que se estaban violando derechos fundamentales contenidos en la Convención Europea debido al rechazo del director de la Fiscalía Pública para otorgar un compromiso para no procesar a su esposo en caso de que él la asistiera para acabar con su vida ${ }^{39}$. El Tribunal convalidó la ley inglesa que no permitía el suicidio asistido. El Tribunal aceptó el argumento de que aunque la señora Pretty no fuera vulnerable, otros enfermos terminales lo eran. La Corte afirmó que la vulnerabilidad de dicho grupo era lo importante. Así las cosas, aunque la señora Pretty tuviese derecho, prima facie, a decidir cómo terminar con su vida, este derecho colisiona con el derecho de los demás a no terminar su vida, y por tanto, el Estado estaba facultado para hacer prevalecer el último. ¿Está justificada tal asimetría?

Estas son las preguntas que necesitamos confrontar. El principio de proporcionalidad, tal y como se plantea, no ofrece recursos para responderlas. Necesitamos una teoría sustantiva para delimitar distinciones cualitativas de este tipo en la ponderación, aun cuando los números y las probabilidades también importen.

PROPORCIONALIDAD Y EL EFECTO HORIZONTAL

DE LOS DERECHOS FUNDAMENTALES

El último ejemplo sobre la necesidad de elaborar teorías sustantivas que sean externas al test de proporcionalidad tiene que ver con la pregunta acerca de si los derechos fundamentales aplican en el ámbito privado y cómo aplican. Aquí hay un debate conocido sobre la posibilidad de interpretar los derechos fundamentales como vinculantes para los particulares, y no solo para las autoridades públicas. ¿Podrían estos derechos tener efectos horizontales, además de los efectos verticales que tradicionalmente se les han otorgado?

En muchas jurisdicciones, la respuesta es afirmativa. Los derechos constitucionales se consideran relevantes para las relaciones entre actores privados. Sin embargo, se han propuesto diferentes teorías sobre las formas en que los derechos producen efectos en el dominio privado. De acuerdo con algunas de ellas, los derechos constitucionales son directamente vinculantes para los particulares. Otras teorías, por el contrario, sostienen que los efectos de dichos derechos son indirectos: cuando el legislador hace leyes que regulan la esfera privada, y cuando los jueces interpretan dichas leyes en un caso particular, deberían tener en cuenta los derechos constitucionales que están en juego ${ }^{40}$.

No es necesario entrar en controversias teóricas en este punto. Lo que interesa para nuestro propósito es lo siguiente: cuando un particular invoca

39 Caso Pretty vs. Reino Unido, 2002. Tribunal Europeo de Derechos Humanos, 423.

40 Una mirada comparativa sobre esta problemática puede encontrarse en $C f r$. The Constitution in Private Relations (ANDRÁs SAJó y RANÁta UitZ eds., 2005). 
un derecho constitucional en contra de otro, bien sea que su efecto horizontal sea directo o indirecto, este último utilizará como defensa su propio derecho a la libertad, u otros derechos fundamentales, como la libertad de asociación, la libertad contractual, la libertad religiosa, entre otros. ¿Cómo pueden decidir los jueces los conflictos entre estos derechos?

El principio de proporcionalidad, naturalmente, entra a hacer parte de este contexto: la restricción de uno de los derechos en favor del otro debe considerarse justificada, si es proporcional. Sin embargo, en este punto es necesario, de nuevo, construir una teoría sustantiva para proveer una guía normativa.

Supongamos que una mujer se queja de discriminación en su contra por razón de género por parte de un empleador, pues no le ha dado un trabajo por ser mujer. Por otra parte, imaginemos que a una mujer a la que no se le permite entrar a un club gastronómico, pues se requiere ser hombre para ser miembro. Si aplicamos el test de proporcionalidad sin un contexto normativo, estaríamos obligados a limitar esta investigación a preguntas como: ¿Qué tan importante es para la primera mujer obtener un trabajo, y para la otra ser admitida en un club? ¿Existirían otras oportunidades abiertas si es rechazada? En otras palabras ¿Cuántos empleadores diferentes podrían ofrecerle a una mujer un trabajo como el que busca, y cuántos clubes gastronómicos podrían recibir a la otra mujer excluida del club exclusivo para hombres? Todos estos factores deben tenerse en cuenta. Sin embargo, necesitamos una teoría más profunda para dar forma a una indagación normativa. El ámbito laboral es diferente al ámbito de las asociaciones, por ejemplo. Las razones que puede invocar un empleador para rechazar un candidato no son idénticas a las que puede dar una asociación. El papel que desempeña el mercado laboral en un orden liberal es tal que la discriminación por razones de género debe ser excluida (excepto en algunas circunstancias especiales). El ámbito de las asociaciones puede ser diferente. Podemos celebrar la diversidad y permitir la proliferación de asociaciones, muy distintas en términos de cuán abiertas son estas. A menos de que tengamos unas bases teóricas que nos ayuden a dar forma a nuestras intuiciones de que los campos del mercado laboral y de las asociaciones son diferentes dentro de un orden liberal, no podremos justificar nuestras conclusiones sobre los efectos horizontales de los derechos de igualdad en los casos particulares ${ }^{41}$.

41 Para una noción de los diversos principios de justicia que gobiernan la distribución de bienes y cargas en diferentes ámbitos de la vida, $c f r$. Michael WALZER, Spheres of Justice (1984). Por ejemplo, en el contexto del constitucionalismo norteamericano, Bruce Ackerman sostiene que el movimiento de los derechos civiles en la década de 1960 condujo a la adopción de leyes que implementaron la "igualdad esférica". Así, se dio una forma diferente a la igualdad en los distintos ámbitos de la vida social. Cfr. 3 Bruce Ackerman, We the People: The Civil Rights Revolution. 127-53 (2014). 
MÁS ALLA DE LA PROPORCIONALIDAD: ALGUNAS

CONSECUENCIAS INSTITUCIONALES

He tratado de demostrar que el principio de proporcionalidad debe estar vinculado con una serie de teorías sustantivas sobre los derechos. Algunas consecuencias institucionales se derivan de esto. Una de las más importantes tiene que ver con el papel de las cortes cuando ejercen el control constitucional.

Si el test de proporcionalidad no necesitara mucha ayuda del tipo de teorías sustantivas que he explorado, parecería ser un test relativamente "mecánico". Los jueces, por supuesto, estarían en desacuerdo cuando aplican este test, pero estos desacuerdos estarían relativamente limitados. Por el contrario, una vez descubrimos que hay un un gran terreno normativo por explorar antes de que se pueda aplicar completamente el test de proporcionalidad, podemos predecir que los desacuerdos entre los jueces serán mayores. Un juez progresista llegará a resultados distintos a los de un juez conservador.

Esto no es nuevo, por supuesto. Después de todo, nos inclinamos a diseñar sistemas que sujetan a los jueces constitucionales a controles democráticos, puesto que somos conscientes de que controversiales doctrinas de moralidad política guiarían, inevitablemente, la jurisprudencia constitucional ${ }^{42}$.

Esto no significa negar la utilidad que en ocasiones tiene el test de proporcionalidad, incluso en ausencia de mayores acuerdos entre los jueces a nivel de teoría normativa sustantiva. En algunos casos, las medidas del gobierno son tan "irracionales" que los jueces pueden desvirtuarlas sin necesidad de ponerse de acuerdo sobre grandes cuestiones. Los jueces pueden utilizar el "método socrático" para exponer las contradicciones argumentativas del gobierno ${ }^{43}$.

En el 2002 en el caso Christine Goodwin v. Reino Unido, por ejemplo, la Corte Europea de Derechos Humanos revisó una ley que no reconoció el nuevo sexo de una persona transgénero. ${ }^{44}$ Curiosamente, el gobierno británico había financiado la cirugía de reasignación de sexo del demandante. La Corte lo percibió como un curso de acción incoherente. No resulta sorpresivo que la Corte no se sorprendiera con el "interés general de la comunidad" que el gobierno invocó para justificar la ley.

42 Así, las consideraciones democráticas explican en parte la tendencia de muchos países europeos para poner el control de constitucionalidad en manos de tribunales constitucionales especializados, en vez de otorgarlo a cortes ordinarias. Cfr. Víctor Ferreres Comella, Constitutional Courts and Democratic Values. A European Perspective 98-108 (2009).

43 Mattias Kumm ha desarrollado un símil interesante entre el papel de los Tribunales cuando deciden demandas en contra del Estado y el papel de Sócrates, que constantemente evaluó la coherencia de las opiniones de los demás. Una corte, según Kumm, responde preguntas y analiza la "coherencia de las respuestas que proporcionan las partes". Cfr. supra nota 12, pp 154-155.

44 Caso Goodwin v. Reino Unido, 2002. Tribunal Europeo de Derechos Humanos, 588. 
En ocasiones, muchas cortes pueden seguir esta estrategia minimalista. Generalmente es una buena idea para los jueces explorar posibilidades de una aproximación minimalista frente a grandes problemas constitucionales ${ }^{45}$. Sin embargo, en muchos casos no hay forma de escapar a controversias profundas, en las que los jueces darán respuestas distintas según sus convicciones morales y políticas. El principio de proporcionalidad no les ofrece soluciones. Es necesaria una teoría sustantiva.

También se afirma con frecuencia que el principio de proporcionalidad es una herramienta que pueden utilizar los jueces para moderar los resultados del proceso legislativo. Las Cortes que se valen del análisis de proporcionalidad "tienden a impulsar los resultados de las políticas al centro de la discusión partidista" ${ }^{46}$. Lo anterior puede ser correcto, pero los jueces necesitan teorías sustantivas para analizar qué tan "extrema" puede ser una medida gubernamental. Por ejemplo, si los jueces consideran un derecho como absoluto, las medidas que el gobierno adopte para restringirlo serán extremas. Si se limita un discurso en particular en virtud de una razón fuera de la lista de razones legítimas, la medida sometida a revisión será extrema. Si una ley prohíbe la discriminación en el campo de las asociaciones exactamente como lo hace una ley que prohíbe la discriminación en el mercado laboral, también se considerará extrema. Por supuesto, si reemplazamos las teorías sustantivas por otras que tienen un contenido diferente, el carácter extremo de las medidas objeto de examen se evaporaran.

Todo lo anterior refuerza la propuesta de que, cuando pensamos en la clase de jueces que queremos para realizar el control constitucional, en el mecanismo para su elección y en los mecanismos de control de su gestión, debemos ser sensibles ante el profundo impacto que las teorías sustantivas necesariamente tienen en la jurisprudencia constitucional. El eje central del principio de proporcionalidad no debería esconder estos puntos. Por el contrario, entendido de forma correcta, este principio nos dirige más allá de sí mismo con frecuencia.

\section{REFERENCIAS}

Ackerman, Bruce. We the People: The Civil Rights Revolution. Cambridge: Harvard University Press, 2014.

Aleinikoff, Alexander. Constitutional Law in the Age of Balancing. 96 Yale Law Journal 943, 48-963, 1987.

Alexy, Robert. A Theory of Constitutional Rights. Oxford; New York: Oxford University Press, 2002.

45 Para una defensa del minimalismo judicial, $c f r$ Cass Sunstein, One Case at a Time: Judicial Minimalism on the Supreme Court (2001).

46 Nota 46. Stone Sweet and Mathews, supra note 2, at 96. 
Alexy, Robert. Formal Principles: Some Replies to Critics. 12 International Journal of Constitutional Law 511, 513, 2014.

Asamblea General de Naciones Unidas. Declaración Universal de los Derechos Humanos. 10 de diciembre de 1948. Res 217 (III) A.

Barak, Aharon. Proportionality. Constitutional Rights and their Limitations. Cambridge: Cambridge University Press, 2012.

BeAtTy, David. The Ultimate Rule of Law. Oxford: Oxford University Press, 2004.

Calabresi, Guido y Bobbit, Philip. Tragic Choices. New York: W.W. Norton, 1978.

Canadian Constitution Act, 1982.

Carta de Derechos Fundamentales de la Unión Europea, 2000 O.J. (C 364).

Cohen-Eliya, Moshe e Porat, Iddo. American Balancing and German Proportionality: The Historical Origins. 8 International Journal of Constitutional Law 263, 271-76, 2010.

Cohen-Eliya, Moshe e Porat, Iddo. Proportionality and the Culture of Justification, 59 American Journal of Comparative Law 463, 2011.

Constitución de Rumania de 1991.

Constitución de Turquía de 1982.

Constitución española de 1978.

Constitución Federal de Suiza de 1999.

Dixon, Rosalind y Landau, David. Transnational Constitutionalism and a Limited Doctrine of Unconstitutional Constitutional Amendments. 13 International Journal of Constitutional Law 606, 2015.

Dworkin, Ronald. Do Liberal Values Conflict? En The Legacy of Isaiah Berlin, MARK Lilla, Ronald Dworkin y Robert B. Silvers (eds.). New York: New York Review Books, 2001.

Ferreres Comella, Víctor. Constitutional Courts and Democratic Values. A European Perspective. New Haven; London: Yale University Press, 2009.

Gardbaum, Stephen. A Democratic Defense of Constitutional Balancing. 4 Law and Ethics Human Rights 78, 2010.

Gardbaum, Stephen. The Myth and the Reality of American Constitutional Exceptionalism, 107 Michigan Law Review 391, 2008.

Gewirt, Alan. Are There Any Absolute Rights? 31 Philadelphia Quarterly 1, 1981.

Grimm, Dieter. Proportionality in Canadian and German Constitutional Jurisprudence. 57 University of Toronto Law Journal 383, 2007.

Huscroft, Grant. Proportionality and the Relevance of Interpretation. En Proportionality and the rule of law: rights, justification, reasoning, Grant Huscroft; BRADLEY W. Miller; GréGoire Webber (eds.). New York, ny: Cambridge University Press, 2014. 
JACKson, VICKI. Constitutional Law in the Age of Proportionality. 124 Yale Law Journal 3094, 2015.

Klatt, Matthias y Meister, Moritz. The Constitutional Structure of Proportionality. Oxford: Oxford University Press, 2012.

Kumm, Mattias. Constitutional Rights as Principles: On the Structure and Domain of Constitutional Justice. 2 International Journal of Constitutional Law 574, 2004.

Kumm, Mattias. The Idea of Socratic Contestation and the Right to Justification: The Point of Rights-Based Proportionality Review. 4 Law and Ethics Human Rights 141, 2010.

Möller, KaI. Proportionality and Rights Inflation. En Proportionality and the rule of law: rights, justification, reasoning, Grant Huscroft; Bradley W. Miller; Grégoire Webber (eds.). New York, NY: Cambridge University Press, 2014.

Möller, KaI. The Global Model of Constitutional Rights. Oxford: Oxford University Press, 2012.

Pilders, Richard H. Avoiding balancing: the role of exclusionary reasons in constitutional law. 45 Hastings Law Journal. 711, 1994.

Sajó, András y Uitz, Ranáta (eds). The Constitution in Private Relations. Eleven International Publishing, 2005.

Schauer, Frederick. A Comment on the Structure of Rights. 27 Georgia Law Review $415,1993$.

Schauer, Frederick. Proportionality and the Question of Weight. En Proportionality and the rule of law: rights, justification, reasoning, Grant Huscroft; Bradley W. Miller; Grégoire Webber (eds.). New York, NY: Cambridge University Press, 2014.

Stone Sweet, Alec y Mathews, Jud. Proportionality Balancing and Global Constitutionalism. 47 Columbia Journal of Transnational Law 72, 2008.

Sunstein, Cass. One Case at a Time: Judicial Minimalism on the Supreme Court. Cambridge: Harvard University Press, 2001.

The Constitution of the Republic of South Africa, 1996.

Tribunal Constitucional de España. STC 22/1984, de 17 de febrero.

Tribunal Europeo de Derechos Humanos. Caso Goodwin vs. Reino Unido, 2002.

Tribunal Europeo de Derechos Humanos. Caso Gough vs. Reino Unido, 2014.

Tribunal Europeo de Derechos Humanos. Caso Pretty vs. Reino Unido, 2002.

Tribunal Europeo de Derechos Humanos. Caso S.A.S vs. Francia, 2014.

Walzer, Michael. Spheres of justice: a defense of pluralism and equality. Oxford: Basil Blackwell, 1989. 